\title{
Automated Bruch's Membrane Opening Segmentation in Cases of Optic Disc Swelling in Combined 2D and 3D SD-OCT Images Using Shape-Prior and Texture Information
}

\author{
Jui-Kai Wang ${ }^{1}$, Randy H. Kardon ${ }^{2,3}$, and Mona K. Garvin ${ }^{2,1}$ \\ 1 Electrical \& Computer Engineering, The University of Iowa, Iowa City, IA, USA \\ 2 Iowa City VA Healthcare System, Iowa City, IA, USA \\ ${ }^{3}$ Ophthalmology \& Visual Sciences, The University of Iowa, Iowa City, IA, USA
}

\begin{abstract}
When the optic disc is swollen, the visibility of the Bruch's membrane opening (BMO) is often drastically reduced in spectral-domain optical coherence tomography (SD-OCT) volumes. Recent work proposed a semi-automated method to segment the BMO using combined information from 2D high-definition raster and 3D volumetric SD-OCT scans; however, manual placement of six landmark points was required. In this work, we propose a fully automated approach to segment the BMO from 2D high-definition and 3D volumetric SD-OCT scans. Using the topographic shape of the internal limiting membrane and textural information near Bruch's membrane, two BMO points are first estimated in the high-definition central B-scan and then registered into the corresponding volumetric scan. Utilizing the information from both the highdefinition BMO estimates and the standard-definition SD-OCT volume, the cost image was created. A graph-based algorithm with soft shapebased constraints is further applied to segment the BMO contour on the SD-OCT en-face image domain. Using a set of 23 volumes with reasonably centered raster scans and swelling larger than $14.42 \mathrm{~mm}^{3}$, the fully automated approach was significantly more accurate than a traditional approach utilizing information only from the SD-OCT volume (RMS error of 7.18 vs. 21.37 in pixels; $p<0.05$ ) and had only a slightly higher (and not significantly different) error than the previously proposed semiautomated approach (RMS error of 7.18 vs. 5.30 in pixels; $p=0.08$ ).
\end{abstract}

\section{Introduction}

In the presence of optic disc swelling due to raised intracranial pressure, recent work has indicated that the shape of Bruch's membrane at the optic nerve head $(\mathrm{ONH})$ as visible from spectral-domain optical coherence tomography (SD-OCT) often deforms towards the vitreous, but that such a deformation does not occur in other causes of optic disc swelling [1]. However, in this initial work [1], quantifying $2 \mathrm{D}$ shape required time-consuming placement of manual landmarks. More recently, as the first step towards a means of quantifying 3D shape, Wang et

X. Chen, M. K. Garvin, J. Liu, E. Trucco, Y. Xu (Eds.): OMIA 2015, Held in Conjunction with MICCAI 2015, Munich, Germany, Iowa Research Online, pp. 33-40, 2015. Available from: http://ir.uiowa.edu/omia/2015_Proceedings/2015/ 
al. [2] proposed a semi-automated approach for segmenting Bruch's membrane opening (BMO) that required six manually placed landmarks.

Building upon the approach of [2], in this work, the goal is to develop a fully automated method to segment BMO in images of optic disc edema using two SD-OCT protocols with different resolutions. In particular, the HD-raster SD-OCT scans provide better visibility of the BMO in 2D but has limited 3D contextual information. On the other hand, the volumetric SD-OCT gives better 3D contextual information but has limited visibility of the BMO region due to the image shadow from the swollen optic disc. The first step of this work is to automatically segment the retinal layers in both OCT images and then register the raster scans to the SD-OCT volume [2]. Next, five candidate BMO sets are individually estimated on the central HD-raster B-scan using the topographic shape of the internal limiting membrane (ILM) and textural information near BM. The mean BMO estimation (computed from the three closest candidates) is then transferred to the volumetric SD-OCT en-face image domain. Finally, a graph-based algorithm with shape-prior information is used to obtain the final BMO contour segmentation from the en-face image. Twenty-three SD-OCT image pairs of optic disc edema are included in this work, and the results from the proposed method are compared to the traditional graph-search method [3], semi-automated method [2] and mean manual tracing from an independent observer.

\section{Methods}

\subsection{Segmentation and Registration for High-Definition and Volumetric SD-OCT Images}

Two ONH-centered SD-OCT protocols (Carl Zeiss Meditec, Dublin, CA) were included in this study: 1) HD 5-line raster protocol, in which each of five B-scans covers $9 \times 2 \mathrm{~mm}^{2}$ with $1024 \times 1024$ pixels, and 2) volumetric $\mathrm{ONH}$ protocol, which covers $6 \times 6 \times 2 \mathrm{~mm}^{3}$ with $200 \times 200 \times 1024$ voxels. First, the ILM and retinal pigment epithelium (RPE) complex were automatically segmented in both HD-raster and volumetric images using a graph-based method $[4,5,2]$ that transforms an image-domain retinal-layer segmentation problem into a graph-domain optimization problem of finding a set of surfaces that forms a minimum-cost closed set. For the purpose of reducing the effect that motion artifacts may cause in the next step of image registration, after obtaining the layer segmentation results, both the HD-raster and volumetric SD-OCT were flattened by realigning the lower surface of the RPE complex as a flat plane [Fig. 1(a),(b)].

After segmenting the retinal layers, the HD-raster scan was automatically registered into the volumetric OCT image using Wang et al.'s approach [2]. With the assumption that the HD-raster central B-scan was centered on the optic disc and knowing the distance between neighboring B-scans $(0.5 \mathrm{~mm} \pm 0.06 \mathrm{~mm})$, the mapped location of each HD-raster B-scan can be limited to a certain number of B-scans in the corresponding volumetric SD-OCT image. Then, considering all five HD-raster B-scans as a group moving together, the summations of the 


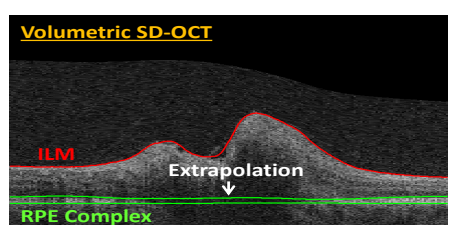

(a)

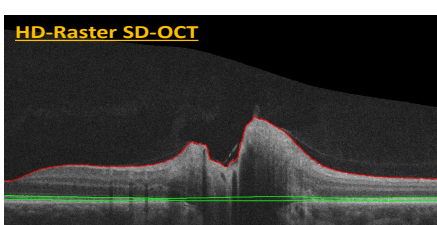

(b)

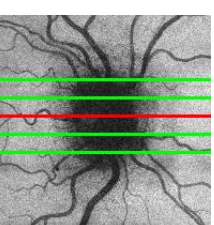

(c)

Fig. 1. Layer segmentation and registration. (a) The central B-scan from a volumetric SD-OCT scan. (b) The central B-scan from the corresponding HD-raster SD-OCT scan. (c) Registration results on the SD-OCT RPE en-face image, where the red and green lines represent the center and the other four HD-raster B-scans, respectively.

five individual 2D registration metrics were computed as a group registration metric.

\subsection{Approximate Estimations of Bruch's Membrane Opening in Central HD-Raster B-scan}

Based on the layer segmentation, five separate estimates of the BMO in the HDraster central B-scans were first computed. Approach 1 used the topographic shape of the ILM. Four other approaches used textural information near Bruch's membrane. Then, the final left and right estimated BMO points on each central B-scan were defined by separately averaging the closest three candidates of the left and right BMO points. Note that the estimated BMO points are used to help create the cost functions in the final BMO segmentation approach (Section 2.3).

Approach 1 By observing a swollen optic disc in the HD-raster central B-scans, it is noticeable that there often exist two ILM peaks that are usually located approximately above the BMO. Because the ILM segmentation is relatively robust, the approximate BMO points can be quickly estimated by detecting the two extreme local peaks on the segmented ILM. [Note: If the HD-raster scan is off center or the optic disc is "severely swollen," the ILM would exhibit only one peak. The algorithm automatically rejects this situation and does not output BMO candidates.]

Approach 2 By averaging the intensities along the $z$-direction of the RPE complex [i.e. the green surfaces in Fig. 1] for every A-scan in the HD-raster central B-scan [Fig. 2(a)], an RPE-projected 1D array (called an RPE-line) was computed. The RPE-line reflects a strong (bright) response at the retinal tissue and a weak (dark) response in the space between the two BMOs, so the left BMO was estimated by detecting the first significant bright-to-dark intensity change on the RPE-line when searching from left to right; the right BMO was detected using the same method but searching the RPE-line from right to left. 
Approach 3 Due to the optic disc swelling, the retinal tissue near the true $\mathrm{BMO}$ region is often obscured by the image shadow so that transitions between the bright and dark segments in the RPE-line may not change sharply enough. Approach 3 used a thresholding method to enhance the contrast between the Bruch's membrane and the image background. The mean intensity of the image background $\left(\mu_{b g}\right)$ was estimated by averaging the pixel intensities of a small region at the top corner of the input HD-raster B-scan. Then, the pixels with intensity value lower than the threshold value $c \cdot \mu_{b g}(c=1.55$ in this work $)$ were thresholded to zero intensity. Next, the thresholded image was rescaled with gray-levels from 0 to 255 . Finally, a $5 \times 3$ mean-image filter was applied to reduce the noise. The output image was denoted as $\mathcal{I}_{T h d}(x, y)$ [Fig. 2(b)]. A different RPE-line was computed using $\mathcal{I}_{T h d}(x, y)$, and the BMO points were estimated from the new RPE-line as in Approach 2.

Approach 4 Textural information helps to distinguish the retinal tissue from the image shadow. A typical method to quantify the textural information is to compute the variance $(\sigma)$ in a sliding window of dimensions $(2 m+1) \times(2 n+1)$ ( $m=n=15$ in this work) throughout the input image. For each pixel $p(x, y)$ in the sliding window, the mean $(\mu)$ can be written as

$$
\mu(x, y)=\frac{1}{(2 m+1) \times(2 n+1)} \sum_{i=-m}^{m} \sum_{j=-n}^{n} p(x+i, y+j) .
$$

Then, the variance image, $\mathcal{I}_{V a r}(x, y)$ [Fig. 2(c)], can be obtained by

$$
\mathcal{I}_{\text {Var }}(x, y)=\frac{1}{(2 m+1) \times(2 n+1)} \sum_{i=-m}^{m} \sum_{j=-n}^{n}[p(x+i, j+j)-\mu(x, y)]^{2} \text {. }
$$

By using $\mathcal{I}_{V a r}(x, y)$, the BMO points were again estimated using the method described in Approach 2.

Approach 5 The last approach is to obtain a gray-level co-occurrence matrix (GLCM) [6] by tabulating the combinations of the intensities for each pixelpair [reference pixel $p(m, n)$ and its neighbor pixel $p(m+d x, n+d y)$ with offset $d=(d x, d y)$. After scaling the input image to $G=16$ gray-levels, GLCMs $\left(M_{\phi}^{(x, y)}[i, j], \phi=45^{\circ}, 90^{\circ}\right.$ and $\left.135^{\circ}\right)$ were computed using the same-sized sliding

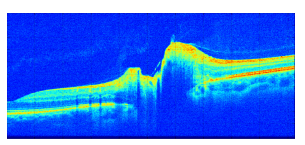

(a)

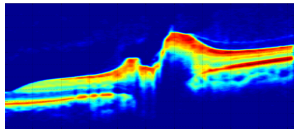

(b)

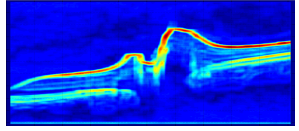

(c)

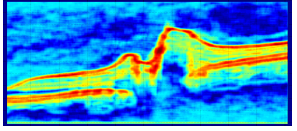

(d)

Fig. 2. HD-raster central image with different image-enhancement methods. (a) Original image. (b) Threshold image $\left[\mathcal{I}_{T h d}(x, y)\right]$. (c) Variance image $\left[\mathcal{I}_{V a r}(x, y)\right]$. (d) GLCM entropy image $\left[\mathcal{I}_{E p y}(x, y)\right]$. Note: Pseudocolor was used for better visualization. 
window from $\mathcal{I}_{V a r}(x, y)$. In order to reflect the orderly information of the current image window at its center pixel $p(x, y)$, the entropy property of the GLCM was adapted. For each $M_{\phi}[i, j]$, the GLCM entropy can be described as

$$
\operatorname{Entropy}_{\phi}(x, y)=\sum_{i, j=0}^{G-1} M_{\phi}^{(x, y)}[i, j] \cdot\left(-\ln M_{\phi}^{(x, y)}[i, j]\right) \text {. }
$$

To achieve the strongest response, the final GLCM entropy image, $\mathcal{I}_{E p y}(x, y)$ [Fig. 2(d)], was described as

$$
\mathcal{I}_{\text {Epy }}(x, y)=\max \left\{\text { Entropy }_{\phi}(x, y) ; \phi=45^{\circ}, 90^{\circ} \text { and } 135^{\circ}\right\} .
$$

The BMO estimation was computed by applying Approach 2 to $\mathcal{I}_{E p y}(x, y)$.

Finally, considering all five sets of the BMO estimations, the final estimated left and right BMO points were decided by averaging the closest three of the five BMO candidates, separately.

\subsection{Bruch's Membrane Opening Segmentation in Volumetric SD-OCT}

A common method to segment the BMO from the SD-OCT en-face view is to transfer the 2D circular object segmentation problem into an (approximately horizontal)-boundary-segmentation problem in the radially unwrapped en-face image domain [3]. In Wang et al.'s work [2], the center for the unwrapped enface image was computed by averaging the six manually-placed BMO points, and the unwrapped cost image was then locally modified by significantly decreasing the cost values at the regions located at these manual landmarks. With the knowledge that the BMO shape is usually elliptical, a graph-search algorithm with a soft constraint to limit the target surface deviating from the shape-prior [7] was used to segment the BMO unwrapped contour.

In this work, the unwrapped center was defined as the middle point of the estimated two BMOs from Section 2.2, and the unwrapped en-face cost image was computed using the same method as Wang et al.'s work [2] without the local modifications. Then, using the the mean and standard deviation BMO sizes in the HD-raster scans reported by Sigler et al. [8], the unwrapped cost image was modified by assigning extremely high costs in regions at least three standard deviations away from the unwrapped mean BMO contour. With this modified cost image, the graph-search algorithm with shape priors was used, and the BMO contour was finally found. (Figures are shown in Section 3.)

\section{Experimental Methods and Results}

Twenty-three subjects with optic disc edema having both volumetric and HDraster SD-OCT image pairs (centered on the optic disc) were included such that all of the volumetric SD-OCT images had a total retinal volume (TRV) larger 
than $14.42 \mathrm{~mm}^{3}$, which is the mean TRV of Frisén grade 2 in [5]. Retinal layers were segmented for both protocol OCT images, and then the HD-raster B-scans were registered into the volumetric scan (Fig. 1). For each HD-raster central scan, five sets of the estimated BMO locations [Fig. 3 (a)] were obtained using the methods from Section 2.2, and the means of the three closest candidates for the left and right points were calculated [Fig. 3 (b)]. The opening locations from the HD-raster central B-scan [pink lines in Fig. 3(b)] were transferred into the SD-OCT RPE en-face domain [pink dots in Fig. 3(c) where the green cross is the halfway point]. Then, the cost image was generated by computing the gradient along the radial direction in the en-face image [Fig. 3 (d), top]. The optic disc size constraints were imposed by assigning extremely high costs (Section 2.3). Next, the 2D graph-based algorithm with the shape-prior constraint was applied, and then the output [red line in Fig. 3(d) bottom] was wrapped back to the RPE en-face image domain [Fig. 3(e)].

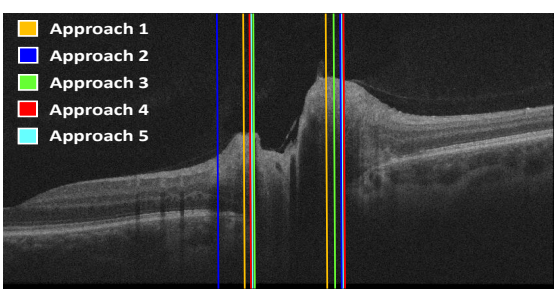

(a)

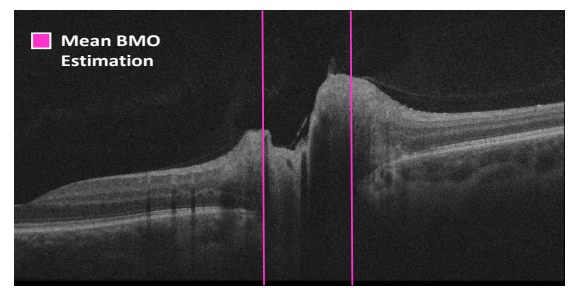

(b)

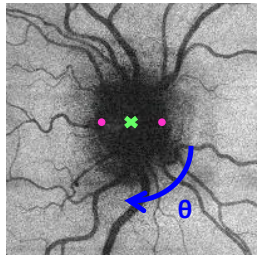

(c)

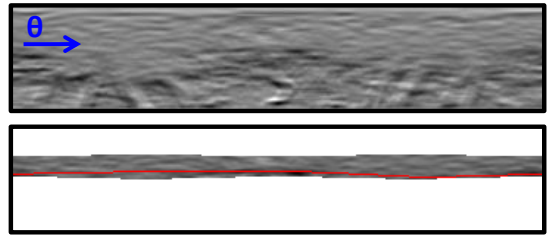

(d)

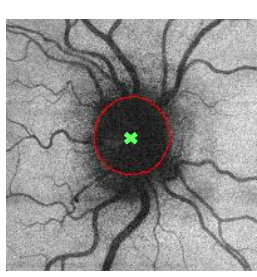

(e)

Fig. 3. BMO estimations from the HD-raster central B-scan to the SD-OCT RPE en-face image. (a) BMO candidates from Section 2.2. (b) Mean BMO estimation. (c) SD-OCT en-face image with registered points (pink dots) and its halfway point (green cross). (d) Top: The cost image in the radial domain; Bottom: Segmentation result on the modified cost image with the constraints of BMO shape and size. (e) BMO segmentation on the en-face image.

For the purpose of validation, we compared the traditional graph-search method [3], semi-automated method [2], and mean manual tracing. For the manual tracings, an independent observer manually placed a BMO landmark in the radially transformed SD-OCT domain at every $30^{\circ}$ (which means 12 manual points per SD-OCT volume) twice. These points were interpolated in the enface view to have a better visualization [2]. Fig. 4 shows a few examples of the qualitative results using the different methods. 


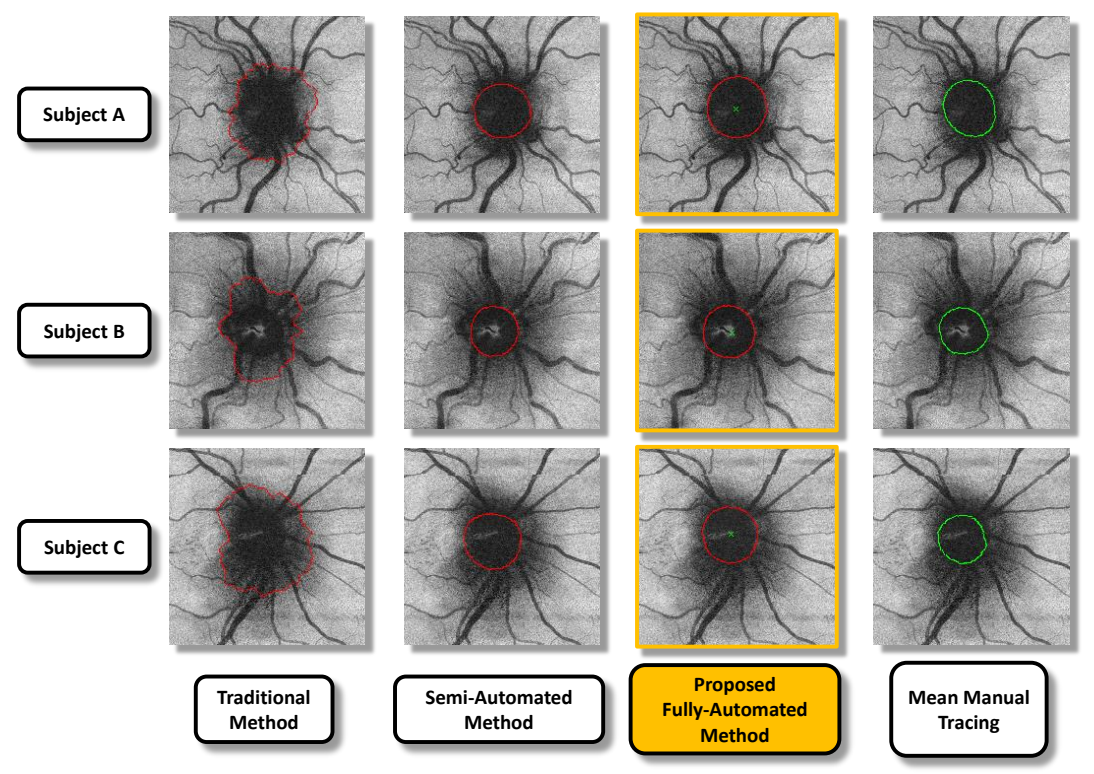

Fig. 4. Comparisons among the traditional method, semi-automated method, proposed fully automated method and mean manual tracing.

For quantitative validation, the signed and root-mean-square (RMS) differences in pixels at every $30^{\circ}$ in the unwrapped en-face domain between the mean manual tracing (MMT) and 1) the traditional method (TM), 2) the semiautomated method (SAM), 3) the proposed fully automated method (FAM) were computed (Table 1). The difference between first and second manual tracing (MT1 and MT2) were also provided as a reference. The signed differences (mean \pm standard deviation) between TM \& MMT, SAM \& MMT, FAM \& MMT and MT1 \& MT2 were $17.83 \pm 8.60,-2.88 \pm 4.53,-0.51 \pm 6.02$ and -0.71 \pm 2.62 , respectively; The RMS differences in the same order were $21.37 \pm 3.89$, $5.30 \pm 3.27,7.18 \pm 3.85$ and $4.33 \pm 2.00$, respectively. The mean RMS difference was significantly smaller using SAM and FAM when compared to TM $(p<0.01)$. Also, the mean RMS difference between SAM \& MMT and FAM \& MMT were not significantly different $(p=0.08)$.

\section{Discussion and Conclusion}

Developing a robust method for directly segmenting the BMO in the volumetric SD-OCT image with a swollen optic disc is extremely challenging. When SDOCT image pairs are centered on the optic disc, with the help from the textural information from the HD-raster central scan and the BMO shape/size-prior, the proposed fully automated method has no significant difference with the semiautomated approach. The limitation is that the proposed method may provide 
Table 1. Summary of signed/RMS BMO positioning differences in pixels ${ }^{\dagger, \ddagger}$.

\begin{tabular}{ccccc} 
& TM \& MMT & SAM \& MMT & FAM \& MMT & MT1 \& MT2 \\
\hline Signed & $17.83 \pm 8.60$ & $-2.88 \pm 4.53$ & $-0.51 \pm 6.02$ & $-0.71 \pm 2.62$ \\
\hline RMS & $21.37 \pm 3.89$ & $5.30 \pm 3.27$ & $7.18 \pm 3.85$ & $4.33 \pm 2.00$ \\
\hline
\end{tabular}

${ }^{\dagger}$ Mean \pm SD in pixels for 23 centralized SD-OCT image pairs with optic disc edema, where the pixel size is $30 \times 30 \mu \mathrm{m}$.

$\ddagger$ TM: Traditional Method; SAM: Semi-Automated Method; FAM: Fully Automated Method; MMT, MT1, MT2: Mean, First and Second Manual Tracing, respectively.

an off-center result when the assumption that the HD-raster scan is centered on the optic disc is violated. Future work is to improve the algorithm such that it has the ability to automatically decide the best optic-disc centered B-scan. Other work could be to provide the BMO segmentation for the original volumetric SDOCT domain instead of the en-face image domain.

Acknowledgments. This study was supported, in part, by the National Institutes of Health R01 EY023279 and the Department of Veterans Affairs Career Development Award 1IK2RX000728.

\section{References}

1. Sibony, P., Kupersmith, M.J., Rohlf, F.J.: Shape analysis of the peripapillary RPE layer in papilledema and ischemic optic neuropathy. Invest Ophthalmol Vis Sci 52(11) (2011) 7987-7995

2. Wang, J.K., Kardon, R.H., Garvin, M.K.: Combined use of high-definition and volumetric optical coherence tomography for the segmentation of neural canal opening in cases of optic nerve edema. In: Proc. SPIE 9413, Medical Imaging 2015: Image Processing. 94133V_1-94133V_9

3. Hu, Z., Abràmoff, M.D., Kwon, Y.H., Lee, K., Garvin, M.K.: Automated segmentation of neural canal opening and optic cup in 3D spectral optical coherence tomography volumes of the optic nerve head. Invest Ophthalmol Vis Sci 51(11) (2010) 5708-5717

4. Garvin, M.K., Abràmoff, M.D., Wu, X., Russell, S.R., Burns, T.L., Sonka, M.: Automated 3-D intraretinal layer segmentation of macular spectral-domain optical coherence tomography images. IEEE Trans Med Imag 28(9) (2009) 1436-47

5. Wang, J.K., Kardon, R.H., Kupersmith, M.J., Garvin, M.K.: Automated quantification of volumetric optic disc swelling in papilledema using spectral-domain optical coherence tomography. Invest Ophthalmol Vis Sci 53(7) (2012) 4069-4075

6. Nailon, W.H.: Texture analysis methods for medical image characterisation. In Mao, Y., ed.: Biomedical Imaging. InTech (2010)

7. Song, Q., Bai, J., Garvin, M.K., Sonka, M., Buatti, J.M., Wu, X.: Optimal multiple surface segmentation with shape and context priors. IEEE Trans Med Imag 32(2) (2013) 376-386

8. Sigler, E.J., Mascarenhas, K.G., Tsai, J.C., Loewen, N.A.: Clinicopathologic correlation of disc and peripapillary region using SD-OCT. Optom Vis Sci 90 (2013) $84-93$ 